\title{
A Converse Lyapunov Theorem for Uncertain Switched Linear Systems
}

\author{
Hai Lin and Panos J. Antsaklis
}

\begin{abstract}
The main contribution of this paper is a converse Lyapunov theorem derived for a class of switched linear systems with time-variant parametric uncertainties. Both discrete-time and continuous-time switched linear systems are investigated. It is shown that the existence of asymptotically stabilizing switching laws implies the existence of a polyhedral Lyapunov function along with conic partition based stabilizing switching laws.
\end{abstract}

\section{INTRODUCTION}

Design stabilizing switching laws for switched systems is one of main research topics in the field of switched systems, and attracts increasing attentions recently, see for example the survey papers [7], [3], the recent books [6], [15] and the references cited therein.

Early efforts for switching stabilization were mainly focused on quadratic stabilization for certain classes of systems. For example, a quadratic stabilization switching law between two LTI systems was considered in [17], in which it was shown that the existence of a stable convex combination of the two subsystem matrices implies the existence of a state-dependent switching rule that stabilizes the switched system along with a quadratic Lyapunov function. A generalization to more than two LTI subsystems was suggested in [13] by using a "min-projection strategy". In [5], it was shown that the stable convex combination condition is also necessary for the quadratic stabilizability of two mode switched LTI system. However, it is only sufficient for switched LTI systems with more than two modes. A necessary and sufficient condition for quadratic stabilizability of switched controller systems was derived in [14]. For robust stabilization, a quadratic stabilizing switching law was designed for polytopic uncertain switched linear systems based on LMI techniques in [19]. All of these methods guarantee stability by using a common quadratic Lyapunov function, which is conservative in the sense that there are switched systems that can be asymptotically (or exponentially) stabilized without using a common quadratic Lyapunov function. There have been some results in the literature that propose constructive synthesis methods to switched systems using multiple Lyapunov functions [3]. For instance, the stabilizing switching law design based on multiple Lyapunov functions was proposed in [16], where piecewise quadratic Lyapunov functions was employed for two mode switched LTI systems. An LMI based method was

The partial support of the National Science Foundation (NSF CCR01$13131)$ is gratefully acknowledged.

H. Lin and P.J. Antsaklis are both with the the Department of Electrical Engineering, University of Notre Dame, Notre Dame, IN 46556, USA. Email: $\{$ hlin1, antsaklis.1\}and. edu proposed in [11] for the stabilizing state-feedback control design of discrete-time piecewise affine systems. Exponential stabilization for switched LTI systems was considered in [12] also based on piecewise quadratic Lyapunov functions, and the synthesis problem was formulated as a bilinear matrix inequality (BMI) problem. Switched nonlinear control systems with actuator constraints and uncertainties were considered in [4]. Notice that these stabilizability conditions, which may be expressed as the feasibility of certain LMIs or BMIs, in the existing literature are basically sufficient only, except for certain cases of quadratic stabilization. A necessary and sufficient condition for asymptotic stabilizability of secondorder switched LTI systems was derived in [18] by detailed vector field analysis.

This paper aims at addressing the complementary problem of switching stabilization, and derive a necessary condition for asymptotic stabilizability of switched linear systems with time-variant parametric uncertainties. In particular, we assume that there exist switching laws to asymptotically stabilize such uncertain switched linear systems, i.e., switching asymptotically stabilizable. The main question studied here is whether there exist (switching control) Lyapunov functions for such switching stabilizable switched linear systems, which is usually referred to as a converse Lyapunov problem.

There are some related work on converse Lyapunov theorem for switched systems. In [2], a converse Lyapunov theorem was derived for the globally uniformly asymptotically stable and locally uniformly exponentially stable switched systems with arbitrary switching signals. It was shown that such arbitrary switching system admits a common Lyapunov function. This converse Lyapunov theorem justifies the common Lyapunov method which was pursued in the literature for arbitrary switching systems. The converse Lyapunov theorem was extended in [10] to switched nonlinear systems that are globally uniformly asymptotically stable with respect to a compact forward invariant set. Notice that both papers are on the existence of a common Lyapunov function for continuous-time switched systems with arbitrary switching signals. Our work differs from theirs in that we are addressing the existence of a (switching control) Lyapunov function for a switching stabilizable switched system, instead of considering a common Lyapunov function for arbitrary switching systems. In addition, we consider robust asymptotic stabilizability for both discrete-time and continuoustime switched linear systems with time-variant parametric uncertainties.

The rest of the paper is organized as follows. In Section II, mathematical models for the discrete-time and continuous- 
time uncertain switched linear system are described, and the converse Lyapunov problem is formulated. In Section III, the converse Lyapunov theorems for discrete-time and continuous-time switching stabilizable uncertain switched linear systems are presented and proved respectively. Finally, concluding remarks are presented.

Notation: The letters $\mathcal{E}, \mathcal{P}, \mathcal{S} \cdots$ denote sets, $\partial \mathcal{P}$ the boundary of set $\mathcal{P}$, and $\operatorname{int}\{\mathcal{P}\}$ its interior. For any real $\lambda \geq 0$, the set $\lambda \mathcal{S}$ is defined as $\{x=\lambda y, y \in \mathcal{S}\}$. The term $\mathrm{C}$-set stands for a convex and compact set containing the origin in its interior.

\section{Problem Formulation}

We consider a collection of discrete-time linear systems described by the difference equations with uncertainties

$x[k+1]=A_{q}(w) x[k], \quad k \in \mathbb{Z}^{+}, \quad q \in Q=\{1, \cdots, N\}$

where $\mathbb{Z}^{+}$stands for non-negative integers.

We also consider a collection of continuous-time linear systems described by the differential equations

$$
\dot{x}(t)=A_{q}(w) x(t), \quad t \in \mathbb{R}^{+}, \quad q \in Q=\{1, \cdots, N\}
$$

where $\mathbb{R}^{+}$denotes non-negative real numbers.

In the above uncertain discrete-time and continuous-time state equations, the state variable $x[k], x(t) \in \mathbb{R}^{n}$. Note that the origin $x_{e}=0$ is an equilibrium for the systems described in (1) and (2). The finite set $Q$ stands for the collection of discrete modes. In particular, for all $q \in Q, A_{q}(w)$ : $\mathcal{W} \rightarrow \mathbb{R}^{n \times n}$, and the entries of $A_{q}(w)$ are assumed to be continuous functions of $w \in \mathcal{W}$, where $\mathcal{W} \subset \mathbb{R}^{v}$ is a given compact set.

Combine the family of discrete-time uncertain linear systems (1) with a class of piecewise constant functions, $\sigma$ : $\mathbb{Z}^{+} \rightarrow Q$, which serves as the switching signal between the collection of discrete-time systems (1). The discrete-time switched linear system can be described as

$$
x[k+1]=A_{\sigma(k)}(w) x[k], \quad k \in \mathbb{Z}^{+}
$$

The signal $\sigma(k)$ is called a switching signal. The particular value of the switching signal $\sigma(k)$ at any given time step $k$ may be generated by a decision-making process. One desirable form of the decision-making process is state feedback based transition law, which can be represented as follows

$$
\sigma(k)=\delta(\sigma(k-1), x[k])
$$

where $\delta: Q \times \mathbb{R}^{n} \rightarrow Q$. The discrete mode is determined by the current continuous state $x[k]$ and the previous mode $\sigma(k-1)$.

Similarly, we introduce a class of piecewise constant functions, $\sigma: \mathbb{R}^{+} \rightarrow Q$, which serves as the switching signal between the class of continuous-time systems (2). The continuous-time switched linear system can be described as

$$
\dot{x}(t)=A_{\sigma(t)}(w) x(t), \quad t \in \mathbb{R}^{+}
$$

and the switching signal is generated by

$$
\sigma(t)=\delta\left(\sigma\left(t^{-}\right), x(t)\right)
$$

where $\delta: Q \times \mathbb{R}^{n} \rightarrow Q$ and $t^{-}=\lim _{\tau \rightarrow 0, \tau>0}(t-\tau)$. The discrete mode is determined by the current continuous state $x(t)$ and the previous mode $\sigma\left(t^{-}\right)$. It is assumed that there are finite switchings within any finite time interval.

For this discrete-time switched system (3)-(4) and continuous-time switched system (5)-(6), we assume that they are asymptotically switching stabilizable, i.e., for any initial condition $x_{0} \in \mathbb{R}^{n}$ there exist switching laws to asymptotically stabilize the switched system. It is known that if there exists a Lyapunov function, usually picked as quadratic or piecewise quadratic, then the switched system can be stabilized. However, the reverse of the statement is not clear yet, that is whether there always exists a (switching control) Lyapunov function for a given switching stabilizable switched system. This is usually called the converse Lyapunov problem.

We focus our attention on this converse Lyapunov problem for both discrete-time switched linear systems (3)-(4) and continuous-time switched linear systems (5)-(6) in this paper, which are formulated as follows.

Problem 1: If there exist switching control laws that make the closed-loop discrete-time switched system (3) globally asymptotically stable, then whether or not we may find a Lyapunov function for the switched system.

Problem 2: If there exist switching control laws that globally asymptotically stabilize the uncertain continuous-time switched linear system (5), then whether or not there exists a Lyapunov function for the switched system.

In the next section, we will derive positive answers for these questions and prove the converse Lyapunov theorems for the asymptotically stabilizable discrete-time and continuous-time switched linear systems respectively.

\section{MAIN RESUlts}

\section{A. Discrete-Time Case}

A converse Lyapunov theorem for the asymptotically stabilizable discrete-time switched linear systems (3)-(4) can be stated as the following theorem.

Theorem 1: If there exist switching control laws that make the closed-loop discrete-time switched system (3) globally asymptotically stable, then there exists a Lyapunov function for the discrete-time switched system (3)-(4).

Proof : First, it is assumed that the switched system can be globally asymptotically stabilized by properly designed switching laws for all initial conditions $x_{0} \in \mathbb{R}^{n}$. In particular, consider the unit sphere, $\mathcal{S}=\left\{x \in \mathbb{R}^{n}:\|x\|=1\right\}$, where $\|\cdot\|$ stands for the Euclidian norm of $\mathbb{R}^{n}$.

For any initial condition $x_{0} \in \mathcal{S}$, there exists a switching signal $\sigma(k)$, by assumption, such that the trajectories starting from $x_{0}$ via following the dynamics

$$
x[k+1]=A_{\sigma(k)}(w) x[k]
$$

asymptotically converge to the origin. Based on the results in [9] and [1] for uncertain linear time-variant systems, there exists a polyhedral $\mathrm{C}$-set $\mathcal{P}_{\sigma}$ such that $x_{0} \in \partial \mathcal{P}_{\sigma}$ and

$$
A_{\sigma(0)}(w) x[0] \in \lambda \mathcal{P}_{\sigma}, \quad \forall w \in \mathcal{W}
$$


where $\lambda$ is a positive scalar and $\lambda<1$.

Consider the Minkowski function of $\mathcal{P}_{\sigma}$, which is defined as

$$
\Psi_{\mathcal{P}_{\sigma}}(x) \doteq \inf \left\{\mu>0: x \in \mu \mathcal{P}_{\sigma}\right\},
$$

we obtain that $\Psi_{\mathcal{P}_{\sigma}}(x[1]) \leq \lambda \Psi_{\mathcal{P}_{\sigma}}(x[0])$, where $x[1]=$ $A_{\sigma(0)}(w) x[0]$ for some $w \in \mathcal{W}$. Note that $\Psi_{\mathcal{P}_{\sigma}}(x[0])=1$, since $x_{0}$ is on the boundary of $\mathcal{P}_{\sigma}$. If we represent the polyhedral C-set $\mathcal{P}_{\sigma}$ canonically as

$$
\mathcal{P}_{\sigma}(x)=\left\{x \in \mathbb{R}^{n}: f_{i} x \leq 1, i=1, \cdots, m\right\},
$$

then the Minkowski function $\Psi_{\mathcal{P}_{\sigma}}(x)$ has the following expression

$$
\Psi_{\mathcal{P}_{\sigma}}(x)=\max _{1 \leq i \leq m}\left\{f_{i} x\right\},
$$

where $f_{i} \in \mathbb{R}^{1 \times n}$ is the gradient vector of facet $F_{i}$ of $\mathcal{P}_{\sigma}$, for $i=1, \cdots, m$.

Assume that for the initial condition $x_{0}, \Psi_{\mathcal{P}_{\sigma}}(x[0])=$ $f_{i_{0}} x[0]$, then $f_{i_{0}} x[1] \leq \Psi_{\mathcal{P}_{\sigma}}(x[1]) \leq \lambda \Psi_{\mathcal{P}_{\sigma}}(x[0])$. Therefore, $f_{i_{0}} x[1] \leq \lambda f_{i_{0}} x[0]$. Let us denote the hyperplane to which the facet $F_{i_{0}}$ is affiliated as $H_{x_{0}}$. The plane $H_{x_{0}}$ can be represented as

$$
H_{x_{0}}=\left\{x \in \mathbb{R}^{n}: f_{i_{0}}\left(x-x_{0}\right)=0\right\},
$$

or

$$
H_{x_{0}}=\left\{x \in \mathbb{R}^{n}: f_{i_{0}} x=1\right\} .
$$

Induced from the plane $H_{x_{0}}$, we define a scalar function $\psi_{H_{x_{0}}}(x)$ as

$$
\psi_{H_{x_{0}}}(x)=\inf \left\{\mu: x \in \mu H_{x_{0}}\right\},
$$

for all the points $x$ in $\mathbb{R}^{n}$. Note that the function $\psi_{H_{x_{0}}}(x)$ is continuous for all $x \in \mathbb{R}^{n}$ and can be represented as an inner product $\psi_{H_{x_{0}}}(x)=f_{i_{0}} x$.

Notice that $\psi_{H_{x_{0}}}\left(A_{q_{0}}(w) x[0]\right) \leq \lambda \psi_{H_{x_{0}}}(x[0])$, where $0<$ $\lambda<1, w \in \mathcal{W}$, and $q_{0}=\sigma(0)$. Following the mode $q_{0}$, the difference of the function value $\psi_{H_{x_{0}}}(\cdot)$ between these two steps is negative, i.e.,

$$
\Delta_{q_{0}}\left(x_{0}\right)=\psi_{H_{x_{0}}}\left(A_{q_{0}}(w) x_{0}\right)-\psi_{H_{x_{0}}}\left(x_{0}\right)<0,
$$

for all $w \in \mathcal{W}$.

Therefore, there exists a positive scalar $0<\epsilon<1-\lambda$, such that

$\Delta_{q_{0}}\left(x_{0}\right)=\psi_{H_{x_{0}}}\left(A_{q_{0}}(w) x_{0}\right)-\psi_{H_{x_{0}}}\left(x_{0}\right)<-\epsilon \psi_{H_{x_{0}}}\left(x_{0}\right)<0$

Note that $\psi_{H_{x_{0}}}\left(x_{0}\right)=1$.

In addition, for any positive scalar $\mu>0$,

$$
\begin{aligned}
\Delta_{q_{0}}\left(\mu x_{0}\right) & =\psi_{H_{x_{0}}}\left(A_{q_{0}}(w) \mu x_{0}\right)-\psi_{H_{x_{0}}}\left(\mu x_{0}\right) \\
& =f_{i_{0}} A_{q_{0}}(w) \mu x_{0}-f_{i_{0}} \mu x_{0} \\
& =\mu\left(f_{i_{0}} A_{q_{0}}(w) x_{0}-f_{i_{0}} x_{0}\right) \\
& =\mu \Delta_{q_{0}}\left(x_{0}\right) \\
& <-\mu \epsilon \psi_{H_{x_{0}}}\left(x_{0}\right) \\
& =-\epsilon \psi_{H_{x_{0}}}\left(\mu x_{0}\right)<0
\end{aligned}
$$

for all $w \in \mathcal{W}$. This implies that all the states on the ray passing through $x_{0}$ will decrease their values of $\psi_{H_{x_{0}}}(x)$ at the next step along the mode $q_{0}$.
Next, because of continuity of the functions $\psi_{H_{x_{0}}}(x)$ and $\Delta_{q_{0}}(x)$, there exists an open neighbor region around $x_{0}$ such that $\Delta_{q_{0}}(x)$ is negative as well. Let us denote such open set as $B_{x_{0}}$. It is always possible to make $B_{x_{0}}$ convex, for example a small ball with $x_{0}$ as center.

In fact, these procedures and negativeness properties can be applied for all the points on $\mathcal{S}$. If we consider all the points on the unit sphere $\mathcal{S}$, then we can derive a collection of similar open sets, which is denoted as $B_{x}$. All these open sets $B_{x}$, for $x \in \mathcal{S}$, represent an open cover of the unit sphere, i.e.,

$$
\bigcup_{x \in \mathcal{S}} B_{x} \supseteq \mathcal{S} \text {. }
$$

Notice that the unit sphere $\mathcal{S}$ is compact, so there exist finite open sets, $B_{x_{i}}$ for $i=1, \cdots, M$, to cover it. Therefore,

$$
\bigcup_{i=1}^{M} B_{x_{i}} \supseteq \mathcal{S}
$$

In the following, we will induce a conic partition of the state space $\mathbb{R}^{n}$ from this finite cover.

Let us consider the region $B_{x_{1}}$ first. For any state $x$ contained inside $B_{x_{1}}$, we have $\Delta_{q_{1}}(x)<0$. This implies that the value of $\psi_{H_{x_{1}}}(x)$ will decrease at the next step, if it follows the mode $q_{1}$ at state $x$. In addition, because of (9), this decreasing property also holds for the smallest conic cone that contains $B_{x_{1}}$, which is denoted as $\mathcal{C}_{1}$. Therefore, we may assign the mode $q_{1}$ to the the conic cone $\mathcal{C}_{1}$.

This procedure can be repeated for all the open sets, $B_{x_{i}}$, for $i=1, \cdots, M$, and induce a finite number of conic cones $\mathcal{C}_{i}$ as well.

Define a scalar

$$
\epsilon_{i}=\inf _{x \in \mathcal{S} \cap B_{x_{i}}} \frac{-\Delta_{q_{i}}(x)}{\psi_{H_{x_{i}}}(x)}>0
$$

Therefore, for all state $x$ contained in $\mathcal{S} \cap B_{x_{i}}$, we have

$$
\begin{aligned}
& \epsilon_{i} \psi_{H_{x_{i}}}(x) \leq-\Delta_{q_{i}}(x) \\
\Rightarrow & \epsilon_{i} \psi_{H_{x_{i}}}(x) \leq \psi_{H_{x_{i}}}(x)-\psi_{H_{x_{i}}}\left(A_{q_{i}}(w) x\right) \\
\Rightarrow & \psi_{H_{x_{i}}}\left(A_{q_{i}}(w) x\right) \leq\left(1-\epsilon_{i}\right) \psi_{H_{x_{i}}}(x)
\end{aligned}
$$

for all $w \in \mathcal{W}$.

It is easy to derive that for all $x \in \mathcal{C}_{i}$,

$$
\psi_{H_{x_{i}}}\left(A_{q_{i}}(w) x\right) \leq\left(1-\epsilon_{i}\right) \psi_{H_{x_{i}}}(x),
$$

which is simplify because of the relationship (9).

It is straightforward to verify that

$$
\bigcup_{i=1}^{M} \mathcal{C}_{i}=\mathbb{R}^{n} .
$$

For each cone $\mathcal{C}_{i}$, draw the hyperplane of $x_{i} \in \mathcal{S}$ as

$$
H_{x_{i}}=\left\{x \in \mathbb{R}^{n}: \psi_{H_{x_{i}}}(x)=1\right\}
$$

Consider the induced half-space

$$
H S_{x_{i}}=\left\{x \in \mathbb{R}^{n}: \psi_{H_{x_{i}}}(x) \leq 1\right\}
$$


The intersection of the finite number of these half-space will define a polyhedral set with the origin in its interior

$$
\mathcal{P}=\bigcap_{i=1}^{M} H S_{x_{i}}
$$

It is easy to prove $\mathcal{P}=\bigcap_{i=1}^{M} H S_{x_{i}}$ is bounded, since otherwise it will lead to a contraction to the fact that the union of the cones $\mathcal{C}_{i}$ is the whole $\mathbb{R}^{n}$. Therefore, the intersection of these half-spaces, i.e., $\mathcal{P}$, is a polyhedron with the origin in its interior, called a polyhedral C-set.

Denote the Minkowski function of the polyhedral C-set $\mathcal{P}$, as $\Psi_{\mathcal{P}}(\cdot)$, which can be expressed as

$$
\Psi_{\mathcal{P}}(x)=\max _{1 \leq i \leq M}\left\{\psi_{H_{x_{i}}}(x)\right\}
$$

The function $\Psi_{\mathcal{P}}(x)$ is piecewise linear continuous function, and has the following properties.

1) Non-negativeness: $\Psi(x) \geq 0, \Psi(x)=0 \Leftrightarrow x=0$;

2) Radius-unboundedness: for any $\mu>0, \Psi(\mu x)=$ $\mu \Psi(x)$

3) Convexity: $\Psi(x+y) \leq \Psi(x)+\Psi(y), \forall x, y \in \mathbb{R}^{n}$.

Basically, $\Psi_{\mathcal{P}}(x)$ defines a distance of $x$ from the origin which is linear in any direction.

In the sequel, we will show that the Minkovski function of the polyhedral $\mathrm{C}$-set $\mathcal{P}$ is a Lyapunov function, which is piecewise linear, for the switched linear systems along with a conic partition based switching law.

We first briefly describe some necessary notations. For the polyhedral C-set $\mathcal{P}$, let $\operatorname{vert}(\mathcal{P})=\left\{v_{1}, v_{2}, \cdots, v_{N}\right\}$ stands for the vertices of a polytope $\mathcal{P}$, while $\operatorname{face}(\mathcal{P})=\left\{F_{1}, F_{2}\right.$, $\left.\cdots, F_{M}\right\}$ denotes its facets. The set of vertices of $F_{i}$ can be found as $\operatorname{vert}\left(F_{i}\right)=\operatorname{vert}(\mathcal{P}) \cap F_{i}$. Finally, we denote the cone generated by the vertices of $F_{i}$ by $\operatorname{cone}\left(F_{i}\right)=\{x \in$ $\left.\mathbb{R}^{n}: \sum_{k} \alpha_{k} v_{i_{k}}, \alpha_{k} \geq 0, v_{i_{k}} \in \operatorname{vert}\left(F_{i}\right)\right\}$. The cone $\left(F_{i}\right)$ has the property that $\forall x \in \operatorname{cone}\left(F_{i}\right)$,

$$
\Psi_{\mathcal{P}}(x)=\psi_{H_{x_{i}}}(x) .
$$

Induced from each facet $F_{i}$ of the polyhedral $\mathrm{C}$-set $\mathcal{P}$, we can get a polyhedral convex cone cone $\left(F_{i}\right)$, which is denoted as $\mathcal{F}_{i}$. Note that $\mathcal{F}_{i} \subseteq C_{i}$ and

$$
\bigcup_{i=1}^{M} \mathcal{F}_{i}=\mathbb{R}^{n} \text {. }
$$

Therefore, we participate the whole state space into a finite number of polyhedral conic cones $\mathcal{F}_{i}$, with mode $q_{i}$ assigned to each cone $\mathcal{F}_{i}$ for $i=1, \cdots, M$. Note that for all the states $x$ contained inside cone $\mathcal{F}_{i}$ we have $\Delta_{q_{i}}(x)<0$.

Next, we introduce a switching law induced from these conic cones and prove that the closed-loop switched system is asymptotically stable.

For such purpose, we define a successor operator, $\operatorname{post}_{q}(\cdot)$, as

$$
\operatorname{post}_{q}(\mathcal{R})=\left\{x^{\prime}: x^{\prime}=A_{q}(w) x ; x \in \mathcal{R}, w \in \mathcal{W}\right\}
$$

which represents all the possible next step states from the current region $\mathcal{R}$ by the transformation $A_{q}(w) x$ for $w \in \mathcal{W}$.
Let us first show some properties of the successor set $\operatorname{post}_{q}(\cdot)$.

Lemma 1: The successor set of a cone is a cone as well. Proof: Denote the cone as $\mathcal{C}$. For any $x \in \operatorname{post}_{q}(\mathcal{C})$, there exist state $x_{0} \in \mathcal{C}$ and $w \in \mathcal{W}$ such that $x=A_{q}(w) x_{0}$. Since $\mathcal{C}$ is a cone, then for any non-negative scalar $\mu, \mu x_{0} \in \mathcal{C}$ and $\mu x=A_{q}(w) \mu x_{0} \in$ post $_{q}(\mathcal{C})$. This implies, $\mu x \in$ post $_{q}(\mathcal{C})$ for any non-negative scalar $\mu$. Hence, $\operatorname{post}_{q}(\mathcal{C})$ is a cone as well.

Note that the successor set of a cone $\mathcal{C}$ may be non-convex. However, a non-convex cone can be written as a finite union of convex cones.

With these preliminary results, we are ready to design a conic partition based switching law under which $\Psi_{\mathcal{P}}(x)$ is monotonically decreasing.

To obtain such conic partition based switching law, we first need to select $x_{i}$ dense enough on $\mathcal{S}$ such that

$$
\bigcup_{i=1}^{M} \operatorname{pre}_{q_{i}}\left(\mathcal{C}_{i}\right) \cap \mathcal{C}_{i}=\mathbb{R}^{n}
$$

where $\operatorname{pre}_{q_{i}}\left(\mathcal{C}_{i}\right)=\left\{x \in \mathbb{R}^{n}:\right.$ post $\left._{q_{i}}(x) \subseteq \mathcal{C}_{i}\right\}$. It is easy to show that pre $_{q_{i}}\left(\mathcal{C}_{i}\right)$ is a convex cone, and so is pre $_{q_{i}}\left(\mathcal{C}_{i}\right) \cap \mathcal{C}_{i}$. Notice that the cones $\mathcal{C}_{i}$ are overlapping each other. The procedure on how to pick $x_{i}$ and expand $\mathcal{C}_{i}$ to satisfy (12) is omitted here due to space limit.

Secondly, For any pair of modes $q_{i}$ and $q_{j}$, if their corresponding cones are adjacent in the sense of

$$
\mathcal{C}_{i} \bigcap \mathcal{F}_{j} \neq \emptyset
$$

then we assume that

$$
\psi_{H_{x_{j}}}(x) \leq \frac{1}{1-\epsilon_{i}} \psi_{H_{x_{i}}}(x)
$$

where $\epsilon_{i}$ is defined before ${ }^{1}$.

Based on the conic cones $\mathcal{F}_{i}$ and the above assumptions, a switching law is proposed as follows.

Assume that the current state $x[k] \in \mathcal{F}_{i}$ and the current discrete mode is $q_{i}$. Consider the following two cases:

If $x[k] \in \mathcal{F}_{i} \cap\left(\right.$ pre $\left._{q_{i}}\left(\mathcal{C}_{i}\right) \cap \mathcal{C}_{i}\right)$, then keep on following the mode $q_{i}$, i.e.,

$$
\sigma(k+1)=\delta(q[k], x[k])=q_{i}
$$

for $x[k] \in \mathcal{F}_{i} \bigcap\left(\right.$ pre $\left._{q_{i}}\left(\mathcal{C}_{i}\right) \cap \mathcal{C}_{i}\right)$.

Otherwise, there must exists at least one $j \neq i$, such that $x[k] \in \mathcal{F}_{i} \bigcap\left(\right.$ pre $\left._{q_{j}}\left(\mathcal{C}_{j}\right) \cap \mathcal{C}_{j}\right)$. This is simply because of the assumption (12). For this case, switch mode to $q_{j}$, i.e.,

$$
\sigma(k+1)=\delta(q[k], x[k])=q_{j},
$$

for $x[k] \in \mathcal{F}_{i} \cap\left(\operatorname{pre}_{q_{j}}\left(\mathcal{C}_{j}\right) \cap \mathcal{C}_{j}\right)$.

In the following, we will show the decreasing of the Lyapunov function $\Psi_{\mathcal{P}}(x[k])$.

\footnotetext{
${ }^{1}$ If we select the pair $x_{i}$ and $-x_{i}$ at the same time, then it is always
} possible to make the function $\psi_{H_{x_{i}}}(x)$ non-negative. 
For the first case, there is no switching occur. Notice that $x[k+1] \in \mathcal{C}_{i}$, since $x[k] \in \operatorname{pre}_{q_{i}}\left(\mathcal{C}_{i}\right)$. Two cases may arise here. First, if $x[k+1]$ is still in $\mathcal{F}_{i}$, then

$\Psi_{\mathcal{P}}(x[k+1])=\psi_{H_{x_{i}}}(x[k+1])<\psi_{H_{x_{i}}}(x[k])=\Psi_{\mathcal{P}}(x[k])$.

Otherwise, $x[k+1]$ falls into another cone $\mathcal{F}_{j}$ adjacent to $\mathcal{C}_{i}$. By the assumption (14), we may obtain that

$$
\begin{aligned}
\Psi_{\mathcal{P}}(x[k+1]) & =\psi_{H_{x_{j}}}(x[k+1]) \leq \frac{1}{1-\epsilon_{i}} \psi_{H_{x_{i}}}(x[k+1]) \\
& <\frac{1}{1-\epsilon_{i}}\left(1-\epsilon_{i}\right) \psi_{H_{x_{i}}}(x[k])=\psi_{H_{x_{i}}}(x[k]) \\
& =\Psi_{\mathcal{P}}(x[k])
\end{aligned}
$$

Secondly, if the mode switches from $q_{i}$ to $q_{j}$ at the time instant $k$, then the next step state $x[k+1]=A_{q_{j}}(w) x[k]$ falls into the cone $\mathcal{C}_{j}$. This is also because of the fact that $x[k] \in \operatorname{pre}_{q_{j}}\left(\mathcal{C}_{j}\right)$. In particular, $x[k+1]$ is contained inside one of the adjacent cone $\mathcal{F}_{j^{\prime}}$, where $j^{\prime}$ may be different from $j$. Anyway, we have

$$
\begin{aligned}
\Psi_{\mathcal{P}}(x[k+1]) & =\psi_{H_{x_{j}}}(x[k+1]) \leq \frac{1}{1-\epsilon_{j}} \psi_{H_{x_{j}}}(x[k+1]) \\
& <\frac{1}{1-\epsilon_{j}}\left(1-\epsilon_{j}\right) \psi_{H_{x_{j}}}(x[k])=\psi_{H_{x_{j}}}(x[k]) \\
& \leq \psi_{H_{x_{i}}}(x[k])=\Psi_{\mathcal{P}}(x[k])
\end{aligned}
$$

The last inequality comes from the definition of $\Psi_{\mathcal{P}}(x)=$ $\max _{1 \leq i \leq M}\left\{\psi_{H_{x_{i}}}(x)\right\}$.

In summary, we have

$$
\Psi_{\mathcal{P}}(x[k+1])<\Psi_{\mathcal{P}}(x[k])
$$

for both cases.

Therefore, we showed that the Minkovski function of the polyhedral C-set $\mathcal{P}, \Psi_{\mathcal{P}}(x[k])$, is monotonically decreasing along the solutions of the switched linear system (3)-(4). Together with other properties of $\Psi_{\mathcal{P}}(x[k]), \Psi_{\mathcal{P}}(x[k])$ serves as a Lyapunov function for the switched system.

As an interesting observation, the existence of an asymptotically stabilizing switching law for the switched system (3)-(4) implies the existence of a conic partition based switching law which globally asymptotically stabilizes the closed-loop switched system. Since a conic partition switching law is a specific class of switching law, we have the following corollary.

Corollary 1: A switched system can be globally asymptotically stabilized by a switching law if and only if it can be stabilized by a conic partition switching law.

\section{B. Continuous-Time Case}

A converse Lyapunov theorem for the robust asymptotic stabilizability of the continuous-time uncertain switched linear systems (5)-(6) can be stated as the following theorem.

Theorem 2: If there exist switching control laws that globally asymptotically stabilize the uncertain continuoustime switched linear system (5), then there exists a Lyapunov function for the switched system (5)-(6).

Proof: The basic idea for the proof of the continuous-time case is quite similar to the discrete-time counterpart. We also construct a conic partition based switching law and a piecewise linear Lyapunov function candidate. The key part is to prove the decreasing of the Lyapunov function candidate along the solutions of the switched system. The decreasing of the Lyapunov function candidate is measured by the negativeness of its Dini derivative along solutions. Mathematically, the Dini derivation, $\mathcal{D}^{+} \Psi(x(t))$, for a continuous function $\Psi$, is defined as

$$
\mathcal{D}^{+} \Psi(x(t))=\lim \sup _{\tau \rightarrow 0, \tau \geq 0} \frac{\Psi(x(t+\tau))-\Psi(x(t))}{\tau} .
$$

Based on the arguments in [1] for linear time variant systems, it can be shown that the Dini derivative of $\Psi$ at the time instant $t$, for $x(t)=x, \sigma(t)=q$, and $w(t)=w$, can be calculated as

$$
\mathcal{D}^{+} \Psi(x(t))=\lim \sup _{\tau \rightarrow 0, \tau \geq 0} \frac{\Psi\left(x+\tau A_{q}(w) x\right)-\Psi(x)}{\tau} .
$$

By assumption, for any initial condition $x_{0} \in \mathcal{S}$, there exists a switching signal $\sigma(t)$ such that the trajectories starting from $x_{0}$ and following the dynamics

$$
\dot{x}(t)=A_{\sigma(t)}(w) x(t)
$$

asymptotically converges to the origin, with $x(0)=x_{0}$. Based on the results in [9] and [1] for uncertain linear timevariant systems, there exists a polyhedral $\mathrm{C}$-set $\mathcal{P}_{\sigma}$ and a positive scalar $\bar{\tau}$ such that $x_{0} \in \partial \mathcal{P}_{\sigma}$ and

$$
\left[I+\tau A_{\sigma(0)}\right](w) x(0) \in \lambda \mathcal{P}_{\sigma}, \quad \forall w \in \mathcal{W}
$$

for all $0<\tau \leq \bar{\tau}$, where $\lambda$ is a positive scalar and less than one.

Consider the Euler approximating system (EAS), which can be represented as

$$
x[k+1]=\left[I+\tau A_{q}(w)\right] x[k],
$$

for some $\tau>0$. The connection of the EAS (17) with its original continuous-time systems is explored by the following lemma.

Lemma 2: [1] If there exists positive scalars $\bar{\tau}>0$ and positive scalar $\lambda<1$ such that $\left[I+\tau A_{\sigma(0)}\right](w) x(0) \in \lambda \mathcal{P}_{\sigma}$ holds for all $0<\tau \leq \bar{\tau}$ and $w \in \mathcal{W}$, then the Dini derivative of $\Psi_{\mathcal{P}_{\sigma}}$ at point $x(0)$ satisfies

$$
\mathcal{D}^{+} \Psi_{\mathcal{P}_{\sigma}}(x(0))<-\beta
$$

where $\beta=\frac{1-\lambda}{\tau}$.

Note that

$$
\Psi_{\mathcal{P}_{\sigma}}(x(0))=\psi_{H_{x_{0}}}\left(x_{0}\right)
$$

while following notations in the previous section. It is easy to derive that for $0<\tau<\bar{\tau}$,

$$
\Delta_{q_{0}}\left(x_{0}\right)=\psi_{H_{x_{0}}}\left(\left[I+\tau A_{q_{0}}(w)\right] x_{0}\right)-\psi_{H_{x_{0}}}\left(x_{0}\right)<0,
$$

for all $w \in \mathcal{W}$. Similar to (9), for any positive scalar $\mu>0$, we have

$$
\Delta_{q_{0}}\left(\mu x_{0}\right)<0
$$


Based on Lemma 2, one obtains that the Dini derivative of $\psi_{H_{x_{0}}}$ for all the states on the ray passing through $x_{0}$ is negative along the mode $q_{0}$.

Also based on continuity and compactness arguments we may derive a finite cover, $B_{x_{i}}$ for $i=1, \cdots, M$,

$$
\bigcup_{i=1}^{M} B_{x_{i}} \supseteq \mathcal{S},
$$

for which the Dini derivative of $\psi_{H_{x_{i}}}$ is negative.

The following steps are to deduce a polyhedral C-set $\mathcal{P}$ and partition the state space $\mathbb{R}^{n}$ into conic cones, $\mathcal{F}_{i}$, which are exactly the same as the discrete-time case.

The switching law induced from the conic partition is

$$
\sigma(t)=\delta(\cdot, x(t))=q_{i}, \quad \text { for } x(t) \in \mathcal{F}_{i}
$$

To show that the Minkowski function of $\mathcal{P}, \Psi_{\mathcal{P}}$, is a Lyapunov function, we need to show the negativeness of its Dini derivative under this switching law. Assume that $x(t) \in \mathcal{F}_{i}$ and current mode $\sigma(t)=q_{i}$. There are two cases to consider.

First, if no switching occurs at $t$, then there exists $\bar{\tau}>0$ such that $\forall 0<\tau \leq \bar{\tau}, x(t+\tau) \in \mathcal{F}_{i}$. Then $\Psi_{\mathcal{P}}(x(t))=$ $\psi_{H_{x_{i}}}(x(t))$ and $\Psi_{\mathcal{P}}(x(t+\tau))=\psi_{H_{x_{i}}}(x(t+\tau))$. Therefore

$\mathcal{D}^{+} \Psi_{\mathcal{P}}(x(t))=\lim \sup _{\tau \rightarrow 0^{+}} \frac{\psi_{H_{x_{i}}}(x(t+\tau))-\psi_{H_{x_{i}}}(x(t))}{\tau}<0$

Else, if switching occurs at time $t$, then there exists $\bar{\tau}>0$ such that $\forall 0<\tau \leq \bar{\tau}, x(t+\tau) \in \mathcal{F}_{j}$ for $j \neq i$. Then $\Psi_{\mathcal{P}}(x(t))=\psi_{H_{x_{i}}}(x(t))=\psi_{H_{x_{j}}}(x(t))$ and $\Psi_{\mathcal{P}}(x(t+\tau))=$ $\psi_{H_{x_{j}}}(x(t+\tau))$. The equality of $\psi_{H_{x_{i}}}(x(t))$ and $\psi_{H_{x_{j}}}(x(t))$ is because switching only occurs at the boundary of $\mathcal{F}_{i}$ and $x(t)$ is at the common boundary between cones $\mathcal{F}_{i}$ and $\mathcal{F}_{j}$. Therefore,

$\mathcal{D}^{+} \Psi_{\mathcal{P}}(x(t))=\lim \sup _{\tau \rightarrow 0^{+}} \frac{\psi_{H_{x_{j}}}(x(t+\tau))-\psi_{H_{x_{j}}}(x(t))}{\tau}<0$.

Therefore, the uncertain switched system (5)-(20) is asymptotic stable and $\Psi_{\mathcal{P}}(x(t))$ serves as a Lyapunov function for the switched system.

Similarly, the existence of an asymptotically stabilizing switching law for the continuous-time switched linear system (5)-(6) implies the existence of a conic partition based stabilizing switching law.

Corollary 2: A continuous-time switched linear system (5)-(6) can be globally asymptotically stabilized by a switching law if and only if it can be stabilized by a conic partition switching law.

\section{CONCluding Remarks}

In this paper, the converse Lyapunov problem for both discrete-time and continuous-time switched linear systems affected by parameter variations were investigated. It was shown here that if the uncertain switched linear systems can be asymptotically stabilized by some switching laws, then there exists a polyhedral Lyapunov function along with conic partition based stabilizing switching laws. An interesting observation from this paper's results is that if a switched linear systems is asymptotically switching stabilizable, then one can always find a stabilizing switching law which is characterized by a conic partition of the state space. The proofs for converse Lyapunov theorems here also proposed constructive methods for constructing such conic partition based stabilizing switching laws. The results presented here could be an important step that leads to a necessary and sufficient condition for stabilizability of switched linear systems. Recently, the switching stabilizability for continuous-time switched linear systems was investigated in [8]. However, the extension to the discrete-time case is challenging.

\section{REFERENCES}

[1] F. Blanchini. Nonquadratic lyapunov functions for robust control. Automatica, 31(3):451-461, 1995.

[2] W. Dayawansa and C. F. Martin. A converse lyapunov theorem for a class of dynamical systems which undergo switching. IEEE Trans. Automat. Contr. 44(4):751-760, 1999.

[3] R. A. Decarlo, M. S. Branicky, S. Pettersson, and B. Lennartson. Perspectives and results on the stability and stabilizability of hybrid systems. In P. J. Antsaklis, editor, Proceedings of the IEEE: Special issue on hybrid systems, volume 88, pages 1069-1082. IEEE Press, 2000.

[4] N. H. El-Farra and P. D. Christofides. Coordinating feedback and switching for control of hybrid nonlinear processes. AIChE Journal, 49:2079-2098, 2003.

[5] E. Feron. Quadratic stabilizability of switched systems via state and output feedback. Technical Report CICS-P-468, MIT, 1996.

[6] D. Liberzon. Switching in Systems and Control. Birkhauser, Boston, 2003.

[7] D. Liberzon and A. S. Morse. Basic problems in stability and design of switched systems. IEEE Contr. Syst. Magazine, 19(5):59-70, 1999.

[8] H. Lin and P. J. Antsaklis. A necessary and sufficient condition for robust asymptotic stabilizability of continuous-time uncertain switched linear systems. In Proc. 43rd IEEE Conf. Decision Control, pages 3690-3695, 2004.

[9] D. Liu and A. Molchanov. Criteria for robust absolute stability of timevarying nonlinear continuous-time systems. Automatica, 38(4):627637, 2002.

[10] J. L. Mancilla-Aguilar and R. A. García. A converse lyapunov theorem for nonlinear switched systems. Systems \& Control Letters, 41(1):6771, 2000.

[11] D. Mignone, G. Ferrari-Trecate, and M. Morari. Stability and stabilization of piecewise affine and hybrid systems: An LMI approach. In Proc. 39th IEEE Conf. Decision Control, pages 504-509, 2000.

[12] S. Pettersson. Synthesis of switched linear systems. In Proc. 42nd IEEE Conf. Decision Control, pages 5283-5288, 2003.

[13] S. Pettersson and B. Lennartson. Stabilization of hybrid systems using a min-projection strategy. In Proc. 2001 American Contr. Conf., pages 223-228, 2001.

[14] E. Skafidas, R. J. Evans, A. V. Savkin, and I. R. Petersen. Stability results for switched controller systems. Automatica, 35(4):553-564, 1999.

[15] Z. Sun and S. S. Ge. Switched Linear Systems: Control and Design. Springer-Verlag, 2005.

[16] M. A. Wicks and R. A. DeCarlo. Solution of coupled lyapunov equations for the stabilization of multi-modal linear systems. In Proc. 1997 American Contr. Conf., pages 1709-1713, 1997.

[17] M. A. Wicks, P. Peleties, and R. A. DeCarlo. Switched controller design for the quadratic stabilization of a pair of unstable linear systems. European J. Control, 4:140-147, 1998.

[18] X. Xu and P. J. Antsaklis. Stabilization of second-order lti switched systems. Inter. J. Contr., 73(14):1261-1279, 2000.

[19] G. Zhai, H. Lin, and P. J. Antsaklis. Quadratic stabilizability of switched linear systems with polytopic uncertainties. Inter. J. Contr., 76(7):747-753, 2003. 